\title{
A New Iris Identification Method Based on Ridgelet Transform
}

\author{
Mojtaba Najafi and Sedigheh Ghofrani
}

\begin{abstract}
Iris recognition system provides an approach for individual identification and is regarded as the sophisticated biometric identification system. Therefore, the exclusive features of iris patterns should be extracted and processed. In this paper, a new feature extraction method according to ridgelet transform for identifying the iris images is provided. At first, after segmentation and normalization the collarette area of iris images has been extracted. Then we improve the quality of image by using median filter, histogram equalization, and the two-dimensional (2-D) Wiener filter as well. Finally, ridgelet transform is employed for extracting features and then, the binary bit stream vector is generated. The Hamming distance (HD) between the input bit stream vector and stored vectors is calculated for iris identification. The experimental results show efficiency of the proposed method.
\end{abstract}

Index Terms - Iris identification, ridgelet transform.

\section{INTRODUCTION}

Biometrics is the technology of identification a person from a physical characteristic and it is based on behavioral characteristics of human structures. Among existing different structural features, it was shown that exclusive features like iris pattern have very suitable features [1]. Some properties of the human iris that enhance its suitability for automatic identification include: 1) its inherent isolation and protection from the external environment, being an internal organ of the eye, behind the cornea and the aqueous humor 2) the impossibility of surgically modifying it without high risk of damaging the user's vision 3) its physiological response to light, which provides the detection of a dead or plastic iris and 4) never are there two irises alike, even for identical twin. Many researchers developed iris recognition systems. Daugman [2] proposed an algorithm which was based on iris codes. Integral differential operators are used to detect the centre and diameter of the iris. The image is converted from cartesian to polar transform and rectangular representation of the region of interest is generated. Feature extraction algorithm uses the complex valued 2-D Gabor wavelets to generate the iris codes which are then matched using HDs. Boles and Boashash [3] used zero crossing point of 1-D wavelet at different levels on same center circle of the iris and pupil centralism. Wildes [4] used Laplace pyramid with four various resolution levels to range and normalize correlation for comparison between input image and database images. Ma et al. [5] used 4 dimensional Haar wavelet analysis in four

Manuscript received November 25, 2012; revised January 28, 2013.

The authors are with the Electrical Engineering Department, Islamic Azad University, South Tehran Branch Tehran, Iran (e-mail: mnajafi.81@gmail.com). steps decomposition. Krichen et al. [6] used wavelet packets to produce an iris code at each level of resolution. Feature extraction may be the most important part, Fig. 1, in any iris recognition systems. Different features were used, a bank of Log- Gabor filters [7], collarete area and daubechies in level 3 [8], [9], A geometrical method for pupil detection was used in [10], contourlet transform [11], wavelet transform [12], estimation of the joint probability of a pair of pixel intensities in predetermined relative positions in the image [13] multichannel Gabor filtering [14], directionlet [15], [16], gabor filter according to phase specifications [17], 1-D coiflet wavelet [18].

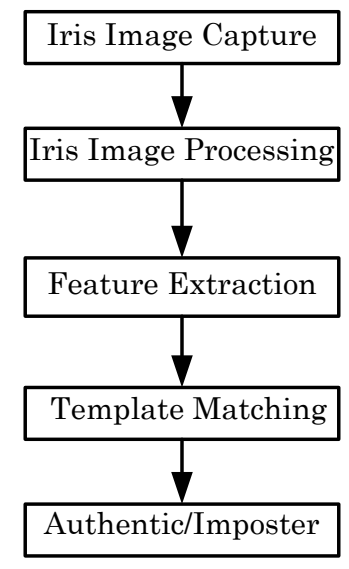

Fig. 1. Stages of iris recognition algorithm

In this paper we propose a new method for feature extraction and compare our results with some mentioned works. Section II deals with image processing steps that include segmentation, normalization, and image quality improvement. Section III deals with ridgelet transform for feature extraction. Section IV deals with our experimental result and finally in section 5 the conclusion of the proposed method is presented.

\section{IRIS IMAGE PROCESSING}

Iris image processing includes three stages, it means segmentation, normalization and contrast enhancement. In addition, for iris processing we need a standard database. We choose the standard CASIA (Chinese Academy of Sciences, Institute of Automation) database. CASIA version 1 iris image database includes 108 different classes, and there are 7 images for each class. There by, we have totally $756(108 \times 7)$ iris images with a resolution of $320 \times 280$ pixels.

\section{A. Segmentation}

The iris is an annular part between pupil (inner boundary) and sclera (outer boundary). It can approximately be 
considered as a circle. The inner and outer iris boundaries should be segmented to provide useful information area for feature extraction. As pupil is black circular region, it is detected easily within the eye image. We use canny edge detector [8] for iris segmentation. Then for binarization, we choose a window with size $(3 \times 3)$ and move it among the segmented image. The color values of the pixel within the window are sub threshold from the window average. If the obtained value for each pixel is less than a given threshold, it will be considered as zero, otherwise set to one. We illustrate the segmented and binarized image in Fig. 2. Now we should find circle contours in iris images by using a practical algorithm to segment iris boundaries. The voting procedure is realized using circular Hough transform in order to search for desired contour from the edge map. Assuming a circle with center coordinate $\left(x_{c}, y_{c}\right)$ and radius $\mathrm{R}$, each edge point on the circle casts a vote in hough space. The circular contour of interest is defined as:

$$
\left(x_{i}-x_{c}\right)^{2}+\left(y_{i}-y_{c}\right)^{2}=R^{2}
$$

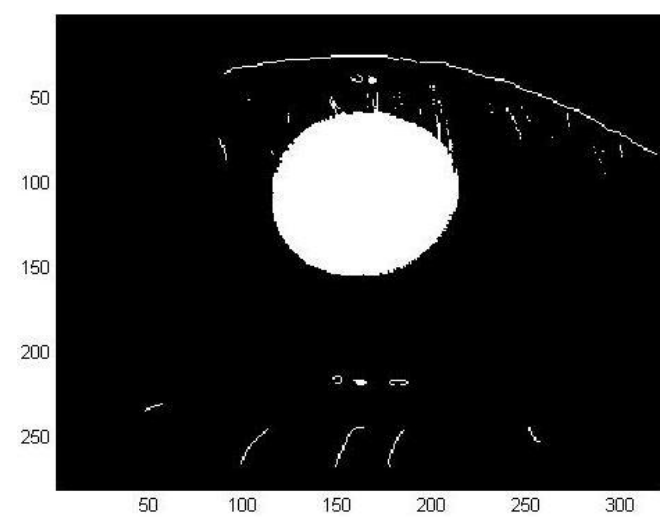

Fig. 2. The segmented and binarized image.

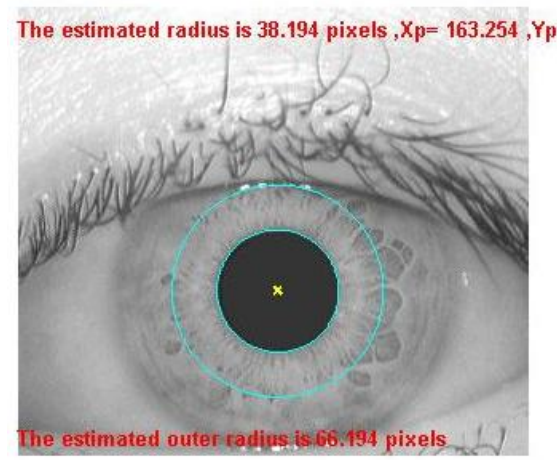

Fig. 3. The inner and outer iris boundaries are shown. The center coordinate is $(163.254,177.734)$, and the estimated inner and outer radius are 38.194 and 66.194 pixels in order.

where $\left(x_{i}, y_{i}\right)$ refers to a point on circle with radius $\mathrm{R}$. The center coordinate and radius of the circle with maximum number of votes are defined as the pupil center and iris inner boundary as well. Considering Hough transformation search the whole eye picture, it takes obviously too much time. Therefore we just search area to the center of image. We know that more complex and rich texture necessary information to identify iris area is close to pupil boundary that named collarette area. The collarette region is less sensitive to the pupil dilation and usually unaffected by the eyelids and the eyelashes. So, after detecting iris inner boundary, the iris outer boundary which has the same center as pupil and predefined radius is specified. Fig. 3 illustrates the inner and outer iris boundaries. The accuracy achieved by the proposed algorithmand four different methods are reported in Table I. As it can be seen, our method achieves the highest accuracy.

\section{B. Normalization}

Iris images that are taken from the same person and in the same place are generally different, so obviously it is true for those captured at different time and different places. Pupil elastic deformation affects the iris size. Due to compensate the iris size variation, iris normalization is an obligation. As the inner and outer boundaries are approximately circle shape, we do iris normalization according to Daugman model [1] which is shown in Fig. 4. According to the Daugman model, a linear mapping has done on each pixel of the iris, and regardless its size and papillary dilation, assigns a pair of real coordinate, $(r, \theta)$. Where $r$ is within the interval $[0,1]$ and $\theta$ is an angle within $[0,2 \pi]$. A normalized iris is shown in Fig. 6. In this paper we present the upper half iris image that probability of eyelids and eyelashes existence is low there. The size of this rectangle is chosen to be $64 \times 256$. Fig. 5 shows the normalized iris image and the windowed respectively.
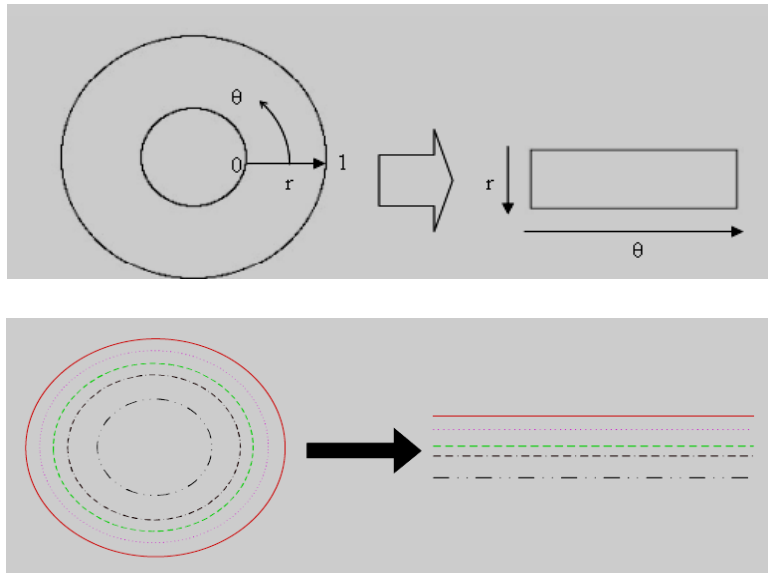

Fig. 4. Iris normalization according to Daugman model [1].

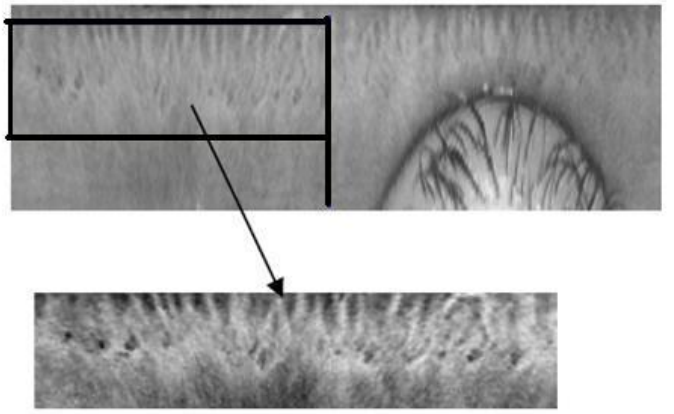

Fig. 5. Normalized iris image (top), cropped special part to get rid of eyelids and eyelashes (bottom).

TABLE I: ACCURACY OF IRIS BOUNDARIES DETECTION.

\begin{tabular}{|l|l|}
\hline Method & Accuracy (\%) \\
\hline Hanho sung [19] & 98.55 \\
\hline Xiaofu [20] & 97.67 \\
\hline Cui et al. [21] & 97.35 \\
\hline Md Slim [22] & 90 \\
\hline Our proposed Method & $\mathbf{9 8 . 6 4}$ \\
\hline
\end{tabular}




\section{Contrast Enhancement}

Iris images should be enhanced before feature extraction. For this purpose we have used median filter, histogram equalization, and the 2-D wiener filter as well. No more description needs for employing median filter and histogram equalization. We just notify that the window size for using the median filter is $(3 \times 3)$. For using the $2-\mathrm{D}$ wiener filter, at first the mean and local variance are computed according to the following equations:

$$
\begin{gathered}
\mu=\frac{1}{M N} \sum_{n_{1}, n_{2} \in \eta} a\left(n_{1}, n_{2}\right) \\
\sigma^{2}=\frac{1}{M N} \sum_{n_{1}, n_{2} \in \eta} a^{2}\left(n_{1}, n_{2}\right)-\mu^{2}
\end{gathered}
$$

where $\eta$ refers to the local window with size $\mathrm{M} \times \mathrm{N}$ around each pixel that is going to process, $\mu$ is the local mean value and $\sigma^{2}$ is the local variance. The output of wiener filter is obtained as follows:

$$
b\left(n_{1}, n_{2}\right)=\mu+\frac{\sigma^{2}-v^{2}}{\sigma^{2}}\left(a\left(n_{1}, n_{2}\right)-\mu\right)
$$

where $v^{2}$ presents the noise variance which is considered to be equal the local mean variance. Fig. 6 shows a contrast enhanced iris image.
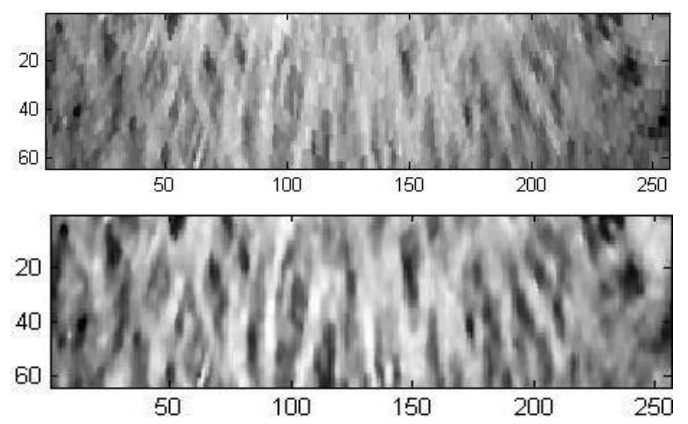

Fig. 6. Normalized iris image (top), enhanced iris image by using median filter, histogram equalization and wiener filter (bottom).

\section{FEATURE EXTRACTION}

It is desirable to explore representation methods which can capture local underlying information in an iris. Here we use ridgelet transform to extract features. The ability of ridgelet transform to make sampling data in different directions, caused that less coefficients need in comparison with using the wavelet transform for feature extraction. In addition we will see that using ridgelet makes the iris identification system accurate and fast.

\section{Ridgelet Transform}

Wavelets are very effective in representing objects with isolated point singularities, while ridgelets are very effective in representing objects with singularities along lines. In fact, one can think of ridgelets as a way of concatenating 1-D wavelets along lines. Hence the motivation for using ridgelets in image processing tasks is appealing since singularities are often joined together along edges or contours in images.
A basic tool for calculating the ridgelet coefficients is to view ridgelet analysis as a form of wavelet analysis in Radon domain. We recall that the Radon transform, Fig. 7, of an object $f$ is the collection of line integrals indexed by $(\theta, t) \in[0,2 \pi] \times R$ given by:

$$
R f(\theta, t)=\int f\left(x_{1}, x_{2}\right) \delta\left(x_{1} \cos \theta+x_{2} \sin \theta-t\right) d x_{1} d x_{2}
$$

where $\delta$ is the Dirac function. The ridgelet coefficients $R_{f}(a, b, \theta)$ of an object $f$ can be obtained as:

$$
R_{f}(a, b, \theta)=\int R f(\theta, t) a^{-\frac{1}{2}} \psi\left(\frac{t-b}{a}\right) d t
$$

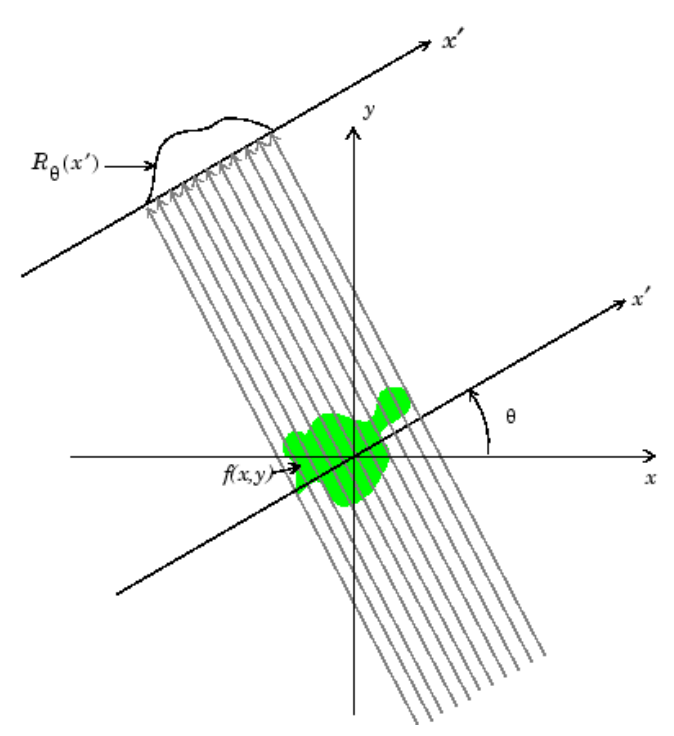

Fig. 7. Radon transform [25].

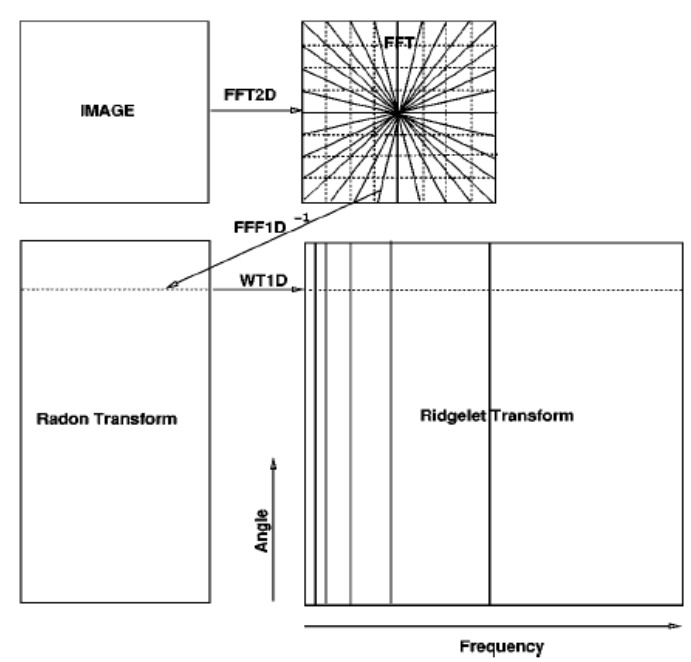

Fig. 8. Ridgelet transform flowgraph. Each of the $2 \mathrm{n}$ radial lines in the Fourier domain is processed separately. The 1-D inverse FFT is calculated along each radial line followed by a 1-D non-orthogonal wavelet transform.In practice, the 1-D wavelet coefficients are directly calculated in the Fourier space [23]

Hence, the ridgelet transform is precisely the application of a 1-D wavelet transform to the slices of the Radon transform where the angular variable $\theta$ is constant and $t$ is varying. Fig. 8 sketches the ridgelet transform flow graph [13]. After calculating the ridgelet coefficients, they should be converted to binary codes. So for $\mathrm{CH}, \mathrm{CV}$, and $\mathrm{CD}$, all coefficients greater than zero are replaced by 1 and all coefficients equal or less than zero are replaced by 0 . In this 
paper we just use $\mathrm{CV}$ and $\mathrm{CH}$ coefficients of ridgelet transform. In Fig. 9, sample binary codes are seen. Thereby we have generated binary codes that experimental results show better performance as an iris classification incomparison with some other different methods.
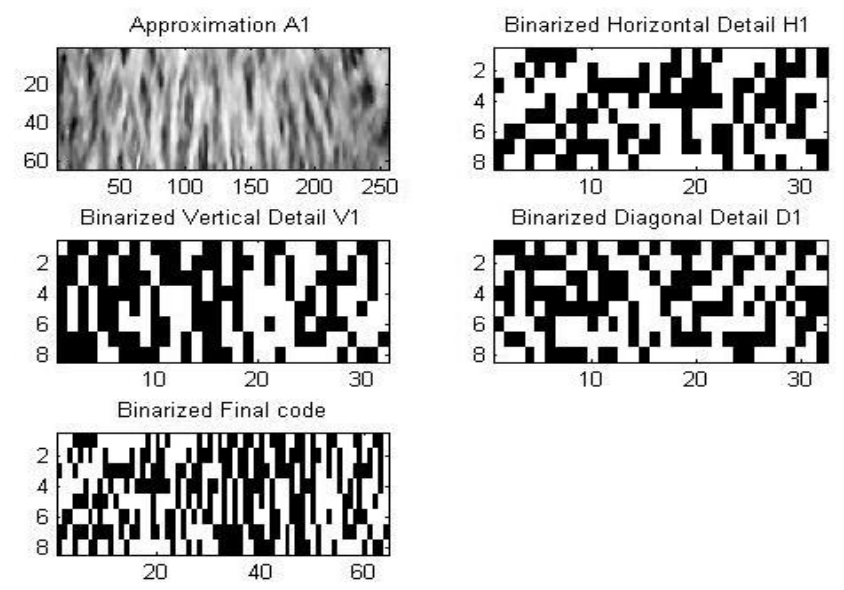

Fig. 9. A sample iris binary code which is generated by the proposed method.

\section{ClassificAtion AND ANALYSIS}

All the obtained features should enter a comparison process to determine the user whose iris photograph was taken. So the last module of an iris recognition system is matching two iris templates. Its purpose is to measure how similar or different templates are and to decide whether they belong to the same individual or not. Although, there are many different methods for this purpose, we choose Hamming distance (HD). The HD between two strings of bits is the number of corresponding bit positions that differ. Using the HD between two bit patterns, a decision can be made as to whether the two patterns were generated from the same iris or from different irises. Because we used the HD as a matching algorithm, we need to have binary feature vectors. Therefore, all feature vectors are digitized before using the HD technique. HD can be made using XOR function as:

$$
H D=\frac{1}{N} \sum_{i=1}^{N} X_{i} \otimes Y_{i}
$$

where $N$ is the number of bits in the feature vector, $X_{i}$ is the $i^{t h}$ feature of the tested iris, and $Y_{i}$, is the $i^{\text {th }}$ feature of the iris template. If two bit patterns are completely independent, such as iris templates generated from different irises, the HD between the two patterns is close to 1 . If two patterns are derived from the same iris, the HD between them is close to 0 . For determining the accuracy of the proposed algorithm, we use Accuracy $=(F A R+F E R) / 2$, where FAR and FER refer to false accept rate and false reject rate [24]. We saw the ridgelet can be implemented based on using different 1-D wavelets. So we have employed different wavelet types for feature extraction and thereby iris identification and reported the accuracy and feature vector length as well as computing time in Table II. In this table, extraction time means the time consuming for segmentation, normalization, enhancement and feature extraction by ridgelet transform. The algorithm has run by using Matlab 7.6, on a Pentium 4, $2.66 \mathrm{MHZ}, 3$ Mb RAM computer. As it can be seen, using Haar as a mother wavelet has the best performance among all selected wavelets. In addition, we have reported the accuracy of our proposed method based on ridgelet transform and some other methods in Table III. We know, wavelet transform extracts coefficients just in 3 directions (horizontal, vertical and diagonal), while ridgelet transform extracts features in more directions. By this method we can sampling coefficients in more and accurate angles in comparison with using the wavelet transform.

TABLE II: RIDGELET TRANSFORM BASED ON USING DIFFERENT 1-D MOTHER WAVELETS.

\begin{tabular}{|l|l|l|l|l|l|l|}
\hline $\begin{array}{l}\text { 1D } \\
\text { Wavelet }\end{array}$ & $\begin{array}{l}\text { Vector } \\
\text { lengh }\end{array}$ & $\begin{array}{l}\text { Total } \\
\text { Error } \\
(\%)\end{array}$ & $\begin{array}{l}\text { Accuracy } \\
(\%)\end{array}$ & $\begin{array}{l}\text { Matching } \\
\text { Time(ms) }\end{array}$ & $\begin{array}{l}\text { Extraction } \\
\text { Time(ms) }\end{array}$ & $\begin{array}{l}\text { Total } \\
\text { Time(ms) }\end{array}$ \\
\hline Harr & 399 & 4.07 & 97.965 & 225.6 & 197 & 422.6 \\
\hline Db1 & 582 & 7 & 96.5 & 299.8 & 200.1 & 499.9 \\
\hline Db2 & 584 & 4.68 & 97.66 & 301.1 & 199.9 & 501 \\
\hline Db3 & 586 & 6.9 & 96.55 & 298.4 & 198.9 & 497.3 \\
\hline Db4 & 586 & 6 & 97 & 300.9 & 198.4 & 499.3 \\
\hline Db5 & 589 & 6.9 & 96.55 & 301.1 & 198.9 & 500 \\
\hline Sym1 & 582 & 6 & 97 & 297.8 & 201.2 & 499 \\
\hline Sym2 & 584 & 4.5 & 97.25 & 299.2 & 201.6 & 500.8 \\
\hline Sym3 & 586 & 6 & 97 & 303.1 & 201.9 & 505 \\
\hline Sym4 & 588 & 5.97 & 97.015 & 297.9 & 202.7 & 500.6 \\
\hline Sym5 & 589 & 5.9 & 97.05 & 301.9 & 200.9 & 502.8 \\
\hline Coif1 & 586 & 4.7 & 97.65 & 299.8 & 201.3 & 501.1 \\
\hline Coif2 & 591 & 7.1 & 96.45 & 301.1 & 200.9 & 502 \\
\hline Coif3 & 596 & 7 & 96.5 & 302.1 & 207.0 & 509.1 \\
\hline Coif4 & 602 & 8 & 96 & 306.4 & 201.1 & 507.5 \\
\hline Coif5 & 607 & 7 & 96.5 & 310.8 & 202.4 & 513.2 \\
\hline
\end{tabular}

TABLE III: THE ACCURACY THAT ACHIEVED By OUR PROPOSED METHOD BASED ON RIDGELET TRANSFORM AND OTHER DIFFERENT METHODS FOR IRIS IDENTIFICATION.

\begin{tabular}{|l|l|}
\hline Method & Accuracy(\%) \\
\hline Amir azizi [11] & 96.5 \\
\hline k. masoud [12] & 95.9 \\
\hline A.T zaim [13] & 95 \\
\hline Li Ma [14] & 94.9 \\
\hline Vladan [15] & 94.7 \\
\hline Hanho Sung [19] & 94.54 \\
\hline Vladan [16] & 94.3 \\
\hline Robert w.Ives [9] & 93 \\
\hline Boles [10] & 92.64 \\
\hline Hao Meng [17] & 87.4 \\
\hline Agus Harjoko [18] & 84.25 \\
\hline Our proposed method & $\mathbf{9 7 . 9 6 5}$ \\
\hline
\end{tabular}

\section{CONCLUSION}

In this paper an effective iris recognition method is proposed which is based on ridgelet transform. We have used different mother wavelet (1-D) in order to implement ridgelet transform. According to our experimental results, the ridgelet transform based on using the Haar as a 1-D mother wavelet has appropriate performance. As the ridgelet transform uses basis function in many directions, employing the ridgelet transform for feature extraction caused our progress to provide shorter binary code and high accuracy as well.

\section{ACKNOWLEDGMENT}

Portions of the research in this paper use the CASIA-IrisV1 database collected by the Chinese Academy of Sciences, Institute of Automation. The authors would like to thank this group for providing an access to the valuable database. 


\section{REFERENCES}

[1] J. Daugman, "High confidence visual recognition by test of statistical independence" IEEE Transactions on Pattern Analysis and MachineIntelligence," vol. 15, pp. 1148-1161, November 1993.

[2] J. G. Daugman, "Statistical richness of visual phase information: Update on recognizing persons by iris patterns," International Journal of Computer Vision, vol. 45, no. 1, pp. 25-38, 2001

[3] W. Boles and B. Boashash, "A Human Identification Technique Using Images of the Iris and Wavelet Transform," IEEE Trans. Signal Processing, vol. 46, no. 4, pp. 1185-1188, 1998.

[4] R. P. Wildes, "Iris Recognition: An Emerging Biometric echnology," Proceedings of the IEEE, vol. 85, no. 9, pp. 1348-1363, 1999.

[5] L. Ma, T. Tan, and Y. Wang, "Iris recognition using circular symmetric filters," International Conference on Pattern Recognition, vol. 2 , pp. $414-417,2002$.

[6] E. Krichen, M. A. Mellakh, S. G. Salicetti, and B. Dorizzi, "Iris identification using wavelet packets," in Proceedings of the 17th International Conference on Pattern Recognition, Cambridge Uk, vol. 4, pp. 335-338, Agust 2004.

[7] R. Zewail, A. Seil, N. Hamdy, and M. Saeb, "Iris Identification Based on Log-Gabor Filtering," in Proceedings of the IEEE Midwest Symposium on Circuits, Systems, \& Computers, vol. 1, pp. 333-336, Dec. 2003

[8] J. Canny, "A Computational Approach to Edge Detection," IEEE Transactions on Pattern Analysis and Machine Intelligence, vol. 8, no. 6, pp. 184-201, Nov 1986.

[9] R. W Ives, "iris recognition using histogram analysis" $38^{\text {th }}$ Asimolar Conference on Signal System and Computer, vol. 1, pp. 562-566, November 2004

[10] W. Boles and B. Boashash, "A Human Identification Technique Using Images of the Iris and Wavelet Transform," IEEE Trans. Signal Processing, vol. 46, no. 4, pp. 1185-1188, 1998

[11] A. Azizi and P. H. Reza, "Efficient iris recognition through improvement of feature extraction and subset selection," International Journal of Computer Science and Information Security, vol. 2, no. 1 June 2009.

[12] K. Masood, "Iris recognition using wavelet," IEEE International Conference on Emerging Technologies, Islamabad, pp. 253-256, November 2007.
[13] A. T. Zaim "A new method for iris recognition using Gary-level coccurance matrix," IEEE Conference on Information and Technology, East Lansing, pp. 350-353, May 2006

[14] L. Ma, Y. H. Wang, and T. N. Tan, "Iris recognition based on multichannel Gabor filtering," in Proceedings of the Fifth Asian Conference on Computer Vision, Australia, pp. 279-283, 2002.

[15] V. Velisavljevic, "Low-complexity iris coding and recognition based on directionlets" IEEE Trans. vol. 4, no. 3, pp. 410-417, Sept. 2009.

[16] V. Velisavljevic, "Low complexity iris recognition with oriented wavelets," $16^{\text {th }}$ IEEE International Conference on Image Processing, Cairo Egypt, pp. 1933-1936, November 2009.

[17] H. Meng and X. Cuiping, "Iris recognition algorithms based on gabor wavelet transform," IEEE International Conference on Mechatronics and Automation, Luoyang henna, pp. 1785-1789, June 2006.

[18] A. Harjoko and S. Hartati, "A method for iris recognition based on 1d coiflet Wavelet," World Academy of Science, Engineering and Technology, vol. 56, pp. 126-129, 2009.

[19] H. Sung and J. lim, "iris localization using collarette boundary localization," IEEE $17^{\text {th }}$ International Conference on Pattern Recognition, Cambridge UK, vol. 4, pp. 857-860, Agust 2004.

[20] X. He and P. Shi, "Anew segmentation approach for iris recognition based on hand-held capture device," Science Direct, Pattern Recognition, vol. 40, issue 4, pp. 1326-1333, April 2007.

[21] R. N. F. Yonh, "An Effective Segmentation Method for Iris Recognition System," $5^{\text {th }}$ IEEE Conference on Visual Information Engineering, Xian China, pp. 548-553, Agust 2008.

[22] M. M. Slim, "Iris recognition:a new approach for iris segmentation," $12^{\text {th }}$ ICCIT (International Conference on Computer and Information Technology, 2009.

[23] J. Starck and J. Emmanuel, "The Curvelet transform for image denoising," IEEE Trans, vol. 11 no. 6, pp. 670-685, June 2002.

[24] M. Vasta and R. Singh, "Reducing false rejection rate of iris recognition using textural and topological features," International Journal of Signal Processing, vol. 2, no. 1, pp. 66-72, 2005.

[25] Math Works - MATLAB and Simulink for Technical Computing. [Online]. Available: http://www.mathwork.com 